\title{
Transport Gap Opening and High On-Off Current Ratio in Trilayer Graphene with Self-Aligned Nanodomain Boundaries
}

Article in ACS Nano - August 2015

DOI: 10.1021/acsnano.5b02877

CITATIONS

0

25 authors, including:

Alexander Chaika

Institute of Solid State Physics RAS

63 PUBLICATIONS 280 CITATIONS

SEE PROFILE

Andrei Varykhalov

Helmholtz-Zentrum Berlin

125 PUBLICATIONS 2,396 CITATIONS

SEE PROFILE
READS

104

D. Marchenko

Helmholtz-Zentrum Berlin

50 PUBLICATIONS $\quad 1,327$ CITATIONS

SEE PROFILE 
Subscriber access provided by TRINITY COLL

\section{Article}

\section{Transport Gap Opening and High On-Off Current Ratio in} Trilayer Graphene with Self-Aligned Nanodomain Boundaries

Han-Chun Wu, Alexander N. Chaika, Tsung-Wei Huang, Askar Syrlybekov, Mourad Abid, Victor Yu. Aristov, Olga V. Molodtsova, Sergey V. Babenkov, D Marchenko, Jaime Sánchez-

Barriga, Partha Sarathi Mandal, Andrei Yu. Varykhalov, Yuran Niu, Barry E. Murphy,

Sergey A. Krasnikov, Olaf Lübben, Jing Jing Wang, Huajun Liu, Li Yang, Hongzhou Zhang,

Mohamed Abid, Yahya T. Janabi, Sergei N. Molotkov, Ching-Ray Chang, and Igor Shvets

ACS Nano, Just Accepted Manuscript • DOI: 10.1021/acsnano.5b02877 • Publication Date (Web): 24 Aug 2015

Downloaded from http://pubs.acs.org on August 25, 2015

\section{Just Accepted}

"Just Accepted" manuscripts have been peer-reviewed and accepted for publication. They are posted online prior to technical editing, formatting for publication and author proofing. The American Chemical Society provides "Just Accepted" as a free service to the research community to expedite the dissemination of scientific material as soon as possible after acceptance. "Just Accepted" manuscripts appear in full in PDF format accompanied by an HTML abstract. "Just Accepted" manuscripts have been fully peer reviewed, but should not be considered the official version of record. They are accessible to all readers and citable by the Digital Object Identifier (DOI®). "Just Accepted" is an optional service offered to authors. Therefore, the "Just Accepted" Web site may not include all articles that will be published in the journal. After a manuscript is technically edited and formatted, it will be removed from the "Just Accepted" Web site and published as an ASAP article. Note that technical editing may introduce minor changes to the manuscript text and/or graphics which could affect content, and all legal disclaimers and ethical guidelines that apply to the journal pertain. ACS cannot be held responsible for errors or consequences arising from the use of information contained in these "Just Accepted" manuscripts. 


\section{Transport Gap Opening and High On-Off Current} Ratio in Trilayer Graphene with Self-Aligned Nanodomain Boundaries

Han-Chun Wu ${ }^{l, *}$, Alexander N. Chaika ${ }^{2,3, *}$, Tsung-Wei Huang ${ }^{4}$, Askar Syrlybekov ${ }^{2}$, Mourad Abid $^{5}$, Victor Yu. Aristov ${ }^{3,6,7}$, Olga V. Molodtsova ${ }^{6}$, Sergey V. Babenkov ${ }^{6}$, D. Marchenko ${ }^{8,9}$, Jaime Sánchez-Barriga ${ }^{8}$, Partha Sarathi Mandal ${ }^{8}$, Andrei Yu. Varykhalov ${ }^{8}$, Yuran Niu ${ }^{10}$, Barry E. Murphy ${ }^{2}$, Sergey A. Krasnikov ${ }^{2}$, Olaf Lübben ${ }^{2}$, Jing Jing Wang ${ }^{2}$, Huajun Liu ${ }^{11}$, Li Yang ${ }^{12}$, Hongzhou Zhang ${ }^{2}$, Mohamed Abid ${ }^{5}$, Yahya T. Janabi ${ }^{13}$, Sergei N. Molotkov ${ }^{3}$, Ching-Ray Chang, and Igor Shvets ${ }^{2}$

${ }^{1}$ School of Physics, Beijing Institute of Technology, Beijing 100081, People's Republic of China ${ }^{2}$ CRANN, School of Physics, Trinity College Dublin, Dublin 2, Ireland

${ }^{3}$ Institute of Solid State Physics RAS, Chernogolovka, Moscow district 142432, Russian Federation

${ }^{4}$ Department of Physics, National Taiwan University, Taipei 10617, Taiwan

${ }^{5}$ KSU-aramco Center, King Saud University, Riyadh 11451, Saudi Arabia

${ }^{6}$ HASYLAB at DESY, D-22607 Hamburg, Germany

${ }^{7}$ Institut für Theoretische Physik, Universität Hamburg, Jungiusstrasse 9, D-20355 Hamburg, Germany

${ }^{8}$ Helmholtz-Zentrum Berlin für Materialien und Energie, D-12489 Berlin, Germany

${ }^{9}$ Freie Universität Berlin, D-14195 Berlin, Germany

${ }^{10}$ MAX-lab, Lund University, Box 118, 22100 Lund, Sweden

${ }^{11}$ Institute of Plasma Physics, Chinese Academy of Sciences, Hefei 230031, People's Republic of China

${ }^{12}$ Electronic Engineering Institute, Hefei 230037, People's Republic of China

${ }^{13}$ Saudi Aramco Materials Performance Unit TSD, Research \& Development Center, Dharhan 31311, Saudi Arabia 
*Address correspondence to:wuhc@bit.edu.cn; chaika@issp.ac.ru
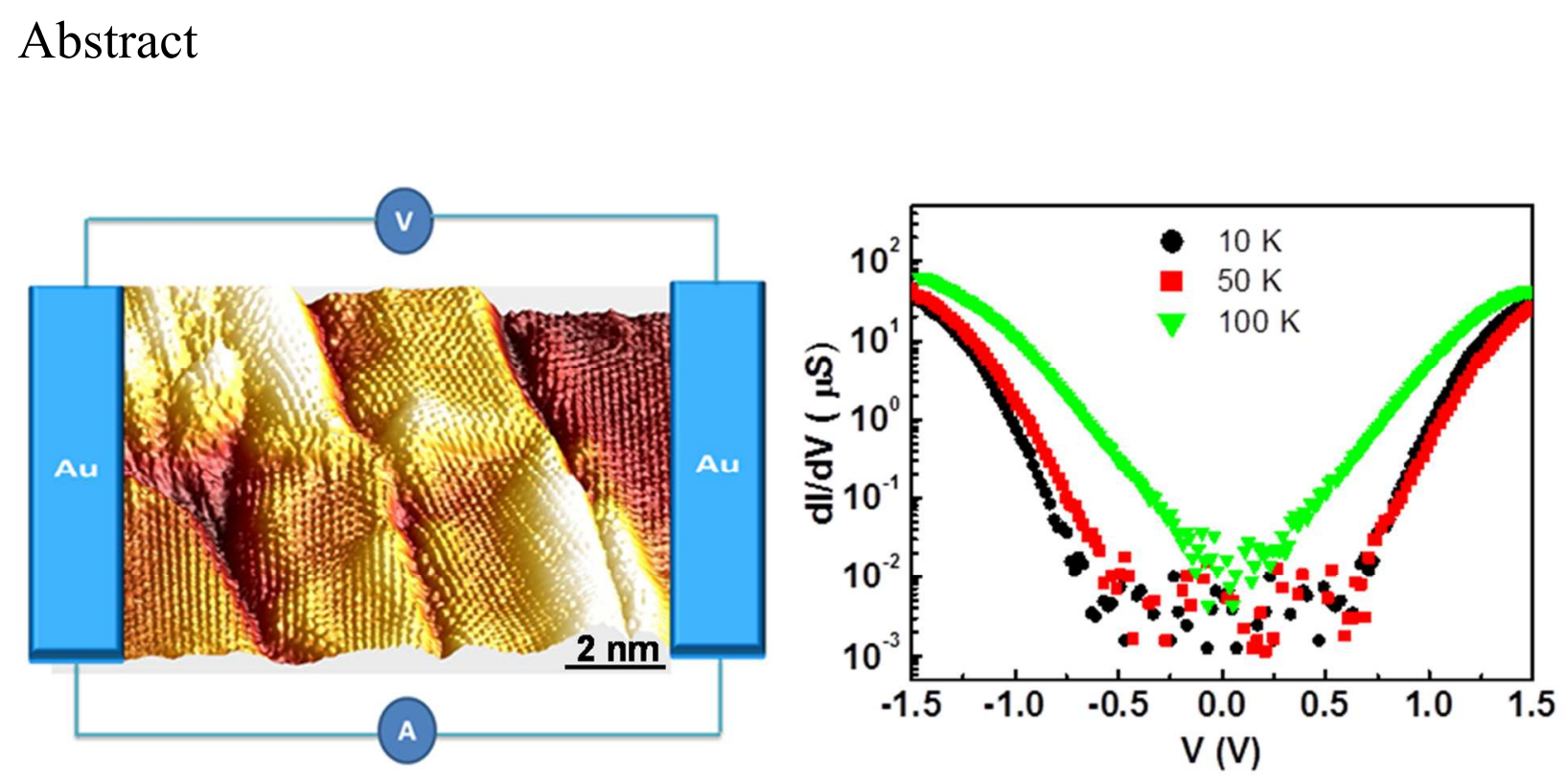

Trilayer graphene exhibits exceptional electronic properties that are of interest both for fundamental science and for technological applications. The ability to achieve a high on-off current ratio is the central question in this field. Here, we propose a simple method to achieve a current on-off ratio of $10^{4}$ by opening a transport gap in Bernal stacked trilayer graphene. We synthesized Bernal stacked trilayer graphene with self-aligned periodic nanodomain boundaries (NBs) on the technologically relevant vicinal cubic-SiC(001) substrate and performed electrical measurements. Our low temperature transport measurements clearly demonstrate that the selfaligned periodic NBs can induce a charge transport gap greater than $1.3 \mathrm{eV}$. More remarkably, the transport gap of $\sim 0.4 \mathrm{eV}$ persists even at $100 \mathrm{~K}$. Our results show the feasibility of creating new electronic nanostructures with high on-off current ratios using graphene on cubic-SiC.

KEYWORDS: trilayer graphene; nanodomain boundary; transport gap; scanning tunneling microscopy; ARPES 
Graphene is a single-atom-thick carbon sheet with extraordinary properties unrivalled by any other known material, ${ }^{1-7}$ which will likely lead to a revolution in many areas of technology. ${ }^{8}$ It displays linear band dispersion, ${ }^{9-10}$ massless Dirac fermions, ${ }^{11}$ and extremely high mobility. ${ }^{12}$ Potentially, graphene-based electronics could consist of just one or a few layers of graphene; however, the absence of a band gap presents a conundrum for the implementation of conventional device architectures, similar to those based on semiconducting materials. ${ }^{13-18}$ Several methods have been proposed for opening band or transport gaps in graphene, such as patterning single-layer graphene into narrow ribbons, ${ }^{19}$ introducing nanoholes into the graphene sheets, ${ }^{20}$ applying a perpendicular electric field, ${ }^{13-18,21-24}$ or applying mechanical strain. ${ }^{25,26}$ Unlike bilayer graphene, gap-opening in trilayer graphene depends on the stacking order of the layers, and notably for ABA (Bernal) stacking it remains metallic even in the presence of a perpendicular electric field. ${ }^{2,21-24}$ Generally, the fabrication, and thus the stacking order, of trilayer graphene devices relies on the mechanical exfoliation of graphite crystals. ${ }^{1}$ Although mechanical exfoliation of graphene from graphite is an effective and successful sample preparation method for fundamental research, it is found that roughly $60 \%$ of trilayer samples prepared this way have a pure ABA stacking order, while the remainder exhibit mixed ABAABC stacking orders. Alternatively, chemical vapor deposition, ${ }^{27,28}$ and vacuum synthesis on silicon carbide surfaces ${ }^{29-32}$ are excellent ways to fabricate large-area few-layer and monolayer graphene. It is known that the graphene produced by these techniques typically contains domainboundaries, ${ }^{30-35}$ which can considerably modify the electronic transport in the graphene. ${ }^{35,36}$ Recent theoretical investigations show that for graphene with self-aligned nanodomain boundaries (NBs), depending on the structure of the NBs, there are two distinct transport behaviors: either high transparency or perfect reflection of charge carriers over large 
energy ranges. ${ }^{36}$ Although this would provide a new way to control the charge carriers without the need to introduce bulk band gaps, there has been no direct experimental evidence so far. The main challenge is to produce graphene with self-aligned periodic NBs.

In this paper, we propose a simple method to synthesize graphene with self-aligned periodic NBs on a semiconducting substrate compatible with silicon technologies and give a clear demonstration of the existence of a transport gap. Our ABA-stacked trilayer graphene with selfaligned periodic NBs was synthesized on $\mathrm{SiC}(001)$ thin films grown on vicinal $\mathrm{Si}(001)$ wafers. Our transport measurements clearly demonstrate that the self-aligned periodic NBs induce a charge transport gap which can reflect charge carriers over a remarkably wide range of energies $(0.4-1.3 \mathrm{eV})$. Moreover, a high on-off current ratio of $10^{4}$ was achieved with a voltage of $0.7 \mathrm{~V}$ below $50 \mathrm{~K}$ and with a voltage of $0.25 \mathrm{~V}$ at $100 \mathrm{~K}$. Our studies pave a way to tailor the transport properties of trilayer graphene.

\section{RESULTS AND DISCUSSION}

For this study, uniform trilayer graphene with a preferential NB direction was fabricated on vicinal $\mathrm{SiC}(001) / \mathrm{Si}(001)$ wafers using $\mathrm{Si}$-atom sublimation followed by high temperature surface graphitization in ultra-high vacuum (UHV). ${ }^{30-32}$ The step direction on the substrate was close to [110], as shown in Figure 1. Raman spectroscopy, scanning tunneling microscopy (STM), low energy electron microscopy (LEEM), low energy electron diffraction (LEED), and angle resolved photoelectron spectroscopy (ARPES) were used to characterize the synthesized trilayer graphene. Figure S1 shows the typical Raman spectrum of graphene on the vicinal SiC substrate measured using a wavelength of $532 \mathrm{~nm}$. The $\mathrm{G}^{\prime}$ or $2 \mathrm{D}$ band can be fitted well with 6 Lorentzians with full widths at half maximum (FWHM) ranging from $21-24.5 \mathrm{~cm}^{-1}$, indicating that the graphene grown is trilayer graphene with NBs present, which is also consistent with the 
electron reflectivity curves measured in different areas of the graphene/SiC(001) sample (Figure S2b). More details about the Raman characterization can be found in the Supporting Information. Figures $2 \mathrm{a}$ and $2 \mathrm{~b}$ show large area STM images of trilayer graphene on the vicinal SiC(001) substrate. Interestingly, our synthesized graphene contains nanometer-scale domains with boundaries elongated in one direction, which is very close to the step direction of the vicinal $\mathrm{SiC}(001)$ sample before graphene synthesis (Figure 1). More remarkably, those nanometer-scale domain structures are continuous even at the intrinsic surface defects (micrometer-sized domain boundaries) as observed in Figure 2a. Therefore, we present a simple method to synthesize graphene with self-aligned periodic NBs. However, LEED characterization suggests that the periodic structures at the nanodomain boundaries between domains are not uniform and straight over the entire sample. The reason for this is the great difficulty in preparing uniform and straight steps for the entire SiC substrate.

Figure 2c shows an atomically resolved STM image containing several nanometer-scale domains connected to each other through the NBs. Detailed analysis of the high resolution STM images measured near the NBs shows that, in most cases, NBs on the vicinal sample (Figures 2c and S3) are rotated by $3.5^{\circ}$ relative to the one of the $<110>$ directions as depicted in Figures 2e and S4. The mechanism responsible this arrangement can be understood as follows. There is a zigzag structure on the left hand side and an armchair structure on right hand side of the NBs (Figure S4a) and the angle on the left side between $\mathrm{Gr}_{\mathrm{L}}$ and the substrate $\mathrm{SiC}$ [110] and the angle on the right side between $\mathrm{Gr}_{\mathrm{R}}$ and $\mathrm{SiC}[110]$ are both $13.5^{\circ}$. This implies that carbon atoms deposited on top of the SiC have equal probability of developing zigzag or armchair structures. Considering the complicated structure near NBs, there are three different graphene structures present: pentagonal, hexagonal, and heptagonal. The internal angle of a pentagon is $108^{\circ}$, a hexagon is 
$120^{\circ}$, and a heptagon is $128.57^{\circ}$. When a pentagon grows next to a hexagon the angle difference is $12^{\circ}$ smaller; while for a heptagon grows next to a hexagon the angle is $8.57^{\circ}$ more. Therefore NBs on top of the SiC deviate from the symmetry of the line by $3.43^{\circ}$, which is consistent with the experimental result of $3.5^{\circ}$. For the left hand side of the NB, a zigzag structure is developed from the NB. This means that two hexagons transform into one pentagon and one heptagon, so the angle difference is $\left(8.57^{\circ}+12^{\circ}\right) / 2=10.28^{\circ}$. For the right hand side of the $\mathrm{NB}$, the armchair structure developed means hexagons must merge with two sides of a pentagon, so the total angle change is $8.57^{\circ} \times 2=17.14^{\circ}$. As Figure $2 \mathrm{e}$ illustrates, this asymmetry near the NBs leads to the formation of a periodic structure along the boundaries, with a period of $1.37 \mathrm{~nm}$. The periodic structure consists of distorted heptagons and pentagons (Figure 2e), which produce the modulations in the atomically resolved STM image measured at the NB (Figure 2d).

In order to extract information about the electronic structure and the stacking order of the nanostructured trilayer graphene synthesized on cubic-SiC(001), we performed ARPES measurements (Figures 3 and S5) and analysed the fine structure of the multiple bands seen near the $\overline{K_{A}}$ and $\overline{K_{B^{\prime}}}$ points in Figure3c. Additionally to gain a better understanding of the experimental data, we performed simulations of photoemission from samples hosting two rotational domains of the trilayer, accounting for the observed momentum splitting $\Delta \mathrm{k}=0.12 \AA^{-1}$. Simulations were performed for the band structure of graphene corresponding to Bernal or Rhombohedral stacking. The band structure of the ABA- and ABC-stacked trilayer graphene acquired within the tight-binding (TB) model was taken from the literature. ${ }^{37}$ The section of trilayer band structure along the direction perpendicular to the $\bar{\Gamma}-\bar{K}$ line of the surface Brillouin zone, which corresponds to the acquisition direction of the experimental dispersion shown in Figure 3c, was simulated by an array of energy distribution curve (EDC) slices composed of the 
Lorentzian peaks residing at the energy positions of the trilayer bands. As ARPES is a non-local method, it sums up photoemission signals from all the various structural phases of graphene at the sample surface. Therefore, the rotational variants of the graphene domains and their rotational displacements were considered in a framework of rotated Brillouin zones and approximated in simulation set by corresponding multiplication and displacement of the model band structure along the momentum axis $K_{\|}$. This approach is illustrated in Figure S6. In particular, photoemission from two principal rotational variants of the trilayer graphene on $\mathrm{SiC}(001)$ were constructed through the superposition of two identical TB band structures of the trilayer displaced by $\pm 0.06 \AA^{-1}$ relative to the $\bar{K}$-point. We assume that intensity of photoemission from the trilayer's bands is proportional to the density of states (DOS) of graphene. Therefore, it was approximated for each trilayer band by a linear function of the binding energy. In fact, there is no noticeable impact of this factor on the results of the simulation (e.g. qualitatively similar results were obtained also for a constant DOS). Conversely, the crucially important factor for agreement or disagreement between the experiment and simulations is the width of the Lorentzian peaks composing EDC slices. In our simulation we varied the FHWM of the Lorentzians between $100 \mathrm{meV}$ and $800 \mathrm{meV}$ to test the correlation between the measured (Figure 3c) and simulated dispersions over energy. Very good correlation between measured ARPES dispersions (Figure 3c) and simulations (Figure 3d) was achieved for a FWHM of the $\pi$-bands as large as $600 \mathrm{meV}$ and for an initial band structure corresponding to the ABA-trilayer. Thus it can be concluded that the stacking order of the trilayer graphene on the cubic-SiC(001) is indeed of ABA-type. Moreover, the $\mathrm{FWHM}=600 \mathrm{meV}$ is three times larger than FWHM measured by ARPES for high-quality quasi-freestanding single layer graphene on hexagonal SiC, Ir or Au. ${ }^{38-40}$ Such significantly enhanced broadening can be ascribed either to 
geometric contributions from a minority of rotational variants distinct from the nanodomains rotated by $\pm 13.5^{\circ}$ from the $<110>$ directions or to quantum scattering of quasiparticles on structural imperfections. Both origins of the broadening are associated with the large number of rotational NBs. The ARPES measurements conducted on the graphene/SiC(001) samples also reveal that the Dirac points are very close to the Fermi level (Figures 3c and S5).This is in full agreement with theoretical simulations for ideal trilayer graphene (Figures 3d and S6) which assume a negligibly small doping level is present in our trilayer graphene.

Figure 4a shows a schematic drawing of a typical graphene nano-gap device. Devices with sub$30 \mathrm{~nm}$ nano-gap contacts were fabricated using standard electron beam lithography techniques. $\mathrm{Ti} / \mathrm{Au}(5 / 45 \mathrm{~nm})$ electrodes were deposited by electron-beam evaporation. The bias voltage was applied perpendicular to the NBs to measure the local transport properties due to the NB defects. Figure $4 \mathrm{~b}$ shows the temperature dependent resistance (R-T) measured with a bias voltage of 0.5 V. The resistance is around $1000 \Omega$ at room temperature and increases significantly with decreasing temperature. Moreover, below $100 \mathrm{~K}$, we cannot detect an appreciable current signal fora bias voltage of $0.5 \mathrm{~V}$, which clearly demonstrates a transport gap in the synthesized trilayer graphene. We fitted the R-T curve with $R(\mathrm{~T}) \approx R_{0} \exp \left(\frac{E_{a}}{\mathrm{~K}_{\mathrm{B}} \mathrm{T}}\right)$, where $\mathrm{R}_{0}$ is the fitting parameter, $K_{B}$ is the Boltzmann constant and $E_{a}$ is the activation energy. The fitting gives an activation energy of $130 \mathrm{meV}$. To further demonstrate the existence of the transport gap, we measured I-V at $10 \mathrm{~K}$ (Figure $4 \mathrm{c}$ ) and plotted the corresponding $\mathrm{dI} / \mathrm{dV}$ in Figure $4 \mathrm{~d}$. Interestingly, for a small bias voltage, we cannot detect any reasonable current signal and the corresponding $\mathrm{dI} / \mathrm{dV}$ is around $0.01 \mu \mathrm{S}$, indicating the existence of a transport gap. The transport gap derived from the $\mathrm{dI} / \mathrm{dV}$ plot is approximately $1.3 \mathrm{eV}$ at $10 \mathrm{~K}$. To understand the effect of the NBs on the electron transport properties, we simulated current across the NBs as a function of gate voltage. In our 
calculations, we used the unit cell as shown in Figure 5a and the gate voltage is adjusted through the on-site energy. A Nonequilibrium Green Function (NEGF) is used and the current is calculated with Landauer-Keldysh formalism. ${ }^{41,42}$ Details about the simulation can be found in the Methods section. Our simulations are summarized in Figure 5b. There is a plateau region where electrons cannot travel, indicating that a gap does open with current driven across the selfaligned NBs and can be tuned with the gate voltage.

Figures $6 \mathrm{a}$ and $6 \mathrm{~b}$ show temperature dependent I-V curves. The transport gap can be clearly observed below $100 \mathrm{~K}$ but disappears at temperatures above $150 \mathrm{~K}$. To obtain the exact value of the transport gap, we plotted the corresponding $\mathrm{dI} / \mathrm{dV}$ curves in Figure $5 \mathrm{c}$ for temperatures below $150 \mathrm{~K}$. Remarkably, the transport gap is approximately the same at $50 \mathrm{~K}$ and $10 \mathrm{~K}$ but substantially lower $(0.4 \mathrm{eV})$ at $100 \mathrm{~K}$. Applying a bias voltage smaller than the transport gap the conductivity of the device is only $10^{-2} \mu \mathrm{S}$, but this increases to $10^{2} \mu \mathrm{S}$ when the bias voltage is larger than the transport gap, which gives a high on-off current ratio of $10^{4}$. Moreover, in our nano-gap contact devices, the NBs are uniform and directed along the step direction on the vicinal substrate (Figure 4a), which gives this system the potential for high-density memory applications. We would like to point out that it was suggested by Huang et al that the scattering rate for a periodic boundary can be written as $<W(k)>=\frac{S^{2} m_{x}}{2 \pi^{2} \hbar^{3}\left|k_{x}\right| d} \times\left[\frac{1-\exp \left(-4 k_{x}^{2} \sigma^{2}\right)}{1+\exp \left(-4 k_{x}^{2} \sigma^{2}\right)-2 \exp \left(-2 k_{x}^{2} \sigma^{2}\right) \cos \left(2 k_{x} d\right)}\right]$, where $d$ is the periodicity along the boundary. Thus, attaining a larger on-off current ratio may result in a reduction in the mobility.

The observed temperature dependence of transport gap could be attributed to two possible mechanisms. ${ }^{25,26,36}$ First, it was suggested by Yazyev and Louie ${ }^{36}$ that a charge transport gap of 
$E_{g}=\hbar v_{F} \frac{2 \pi}{3 d} \approx \frac{1.38}{d(\mathrm{~nm})}(\mathrm{eV})$ can be formed by a non-symmetric NB associated with a lattice mismatch at the boundary line, where $\hbar$ is the reduced Planck's constant, $v_{F}$ is the Fermi velocity. As indicated in Figures $2 \mathrm{~d}$ and $2 \mathrm{e}$, the asymmetric rotation of the graphene lattices in the neighbouring domains relative to the NB leads to a $1.37 \mathrm{~nm}$ periodicity along the NB. According to the theory, ${ }^{36}$ this periodicity should produce a transport gap of approximately $1.0 \mathrm{eV}$, which is consistent with our transport measurements. Therefore, our electrical and STM characterizations support the explanation of the transport gap being based on the Yazyev and Louie theory of asymmetrically rotated domains ${ }^{36}$ for the Bernal-stacked trilayer graphene with the NBs oriented close to the [110] direction (Figure 2e). The disappearance of the transport gap at temperatures above $150 \mathrm{~K}$ in this case can be related to the presence of defects in the graphene trilayer. It is known that defects can remarkably modify the transport properties of graphite and graphene. ${ }^{43}$ There are two kinds of defects in our trilayer graphene: defects at the NBs and interstitial defects in subsurface layers. In STM experiments (Figures 2c and 2d) and Raman characterization (Figure S1), we found that defects at the NBs are stable even at room temperature. Thus they should not have a pronounced impact on the temperature dependence of the trilayer graphene's conductivity (Figures 4 and 6). However, the stability of interstitial defects in subsurface layers (Figure 6d) is temperature dependent. In graphite, the migration activation energy of interstitials, which is usually below $0.03 \mathrm{eV},{ }^{44-47}$ depends on the presence of domain boundaries and the stress in the layer. In our trilayer graphene on vicinal $\mathrm{SiC}$, defects and interstitials may exist which may be frozen under $100 \mathrm{~K}$ and migrate at higher temperatures $(100-300 \mathrm{~K}){ }^{44}$ The mobility of such interstitial defects could be responsible for the disappearance of the transport gap at temperatures above $100 \mathrm{~K}$ (Figure 6). 
Another possible origin of the transport gap observed in trilayer graphene on vicinal $\mathrm{SiC}(001)$ at temperatures below $150 \mathrm{~K}$ could be related to mechanical strain applied to the graphene nanoribbons during the sample cooling. It was shown that applying a uniaxial mechanical strain to bilayer graphene $\mathrm{e}^{26}$ or graphene nanoribbon ${ }^{25}$ can provide a band gap of the order of 0.2-0.4 eV which is also in agreement with the electrical measurements (Figure 6). For our graphene grown on vicinal $\mathrm{SiC}$, to relieve the in-plane compressive strain, ripples can be formed at asymmetric NB. ${ }^{36}$ Indeed, Figures $2 \mathrm{c}, 2 \mathrm{~d}$ and S3 show that the boundaries of all the individual domains on the $\mathrm{SiC}(001)$ do possess such a rippled morphology. As shown in our previous work ${ }^{30}$, the graphene is quasi-freestanding on the $\mathrm{SiC}$, with only some parts of the layers (usually, NBs) supported by the substrate. The thermal expansion coefficients and their temperature dependence are remarkably different for $\mathrm{SiC}^{48}$ and both micrometer-sized and nanostructured graphene, ${ }^{49-51}$ which can induce in-plane strain and lead to the ripple formation during sample cooling after high temperature graphene synthesis.

To clarify the origin of the charge transport gap, we measured I-V at $10 \mathrm{~K}$ with the current applied along the boundaries (Figure 7a). No transport gap is observed and the I-V curve displays nonlinear behavior. This indicates that the observed charge transport gap for current across the NBs is mainly due to the self-aligned periodic NBs which can reflect charge carriers over a range of energies. It also rules out the effect of the contact resistance between graphene and electrode. In addition to this, we also conducted photoemission experiments on the vicinal $\mathrm{SiC}(001)$ samples at temperatures between $100 \mathrm{~K}$ and $300 \mathrm{~K}$ (Figure 7b). Our experimental data undoubtedly show that the valence band edge position does not change with decreasing temperature proving that the observed transport gap is not related to a band gap opening during the sample cooling. At the same time, the secondary electron cutoff (SEC) measurements reveal 
a change of the work function between $150 \mathrm{~K}$ and $100 \mathrm{~K}$ of $\sim 0.36 \mathrm{eV}$, i.e., the values observed in the transport measurements (Figure 6c). Thus, both I-V characterization and low temperature photoemission measurements lead us to conclude that the observed charge transport gap most probably should be attributed to the self-aligned periodic NBs.

\section{CONCLUSION}

In summary, we have presented transport studies on trilayer graphene synthesized on vicinal cubic-SiC(001) substrates. Our electrical measurements clearly demonstrate the opening of a transport gap in the nanostructured graphene synthesized on this stepped surface. Remarkably, the effect can be observed even at $100 \mathrm{~K}$ and produces a current on-off ratio of $10^{4}$. This transport gap was notably observed in the Bernal stacked trilayer graphene. This behavior shows that it is possible to create new tunable electronic nanostructures with graphene on cubic $\mathrm{SiC}$, thus creating opportunities for a wide range of new electronic applications.

\section{Methods}

Uniform trilayer graphene was fabricated on $\mathrm{SiC}(001)$ thin films, grown on vicinal $\left(2^{\circ}\right.$ miscut $)$ $\mathrm{Si}(001)$ wafers, using Si-atom sublimation followed by high temperature surface graphitization in the UHV preparation chamber of a room temperature (RT) GPI-300 scanning tunneling microscope. ${ }^{30,31}$ The base pressure in the analytical chamber was in the range of $4-6 \times 10^{-11}$ mbar. It did not exceed $2 \times 10^{-10}$ mbar during the direct current heating of the $3 \times 8 \times 0.5 \mathrm{~mm}^{3}$ $\mathrm{SiC}(001) / \mathrm{Si}(001)$ samples at temperatures of $1300-1350^{\circ} \mathrm{C}$ and rapidly recovered after the thermal cycles. The graphene synthesis included an UHV deposition of several monolayers of silicon atoms onto the clean, carbon-rich $\mathrm{SiC}(001)-1 \times 1$ surface and annealing at gradually increasing substrate temperatures in the range of $700-1300^{\circ} \mathrm{C}$ until the carbon-rich $\mathrm{c}(2 \times 2)$ 
reconstruction was achieved. The typical duration of each annealing step leading to the consecutively assembly of the $(3 \times 2),(5 \times 2), c(4 \times 2),(2 \times 1)$, and $c(2 \times 2)$ reconstructions was in the range of 10-30 minutes depending on the temperature applied and the pressure in the UHV chamber. Then, flash heating $\left(10-20\right.$ seconds) at $1350^{\circ} \mathrm{C}$ with post-annealing at $600-700^{\circ} \mathrm{C}$ were used to convert the $\mathrm{c}(2 \times 2)$ surface reconstruction into honeycomb lattice. Full details of the graphene synthesis and step-by-step STM studies of the SiC(001) surface atomic structure during synthesis have been described elsewhere. ${ }^{31}$ The principal difference between the current graphene fabrication procedure and previously reported works ${ }^{30,31}$ is the utilization of the vicinal (stepped) $\mathrm{SiC}(001) / \mathrm{Si}(001)$ substrate.

The growth of few-layer graphene on cubic-SiC(001) in UHV at pressure below $2 \times 10^{-10} \mathrm{mbar}$ is self-limiting at three graphene layers. ${ }^{30,31}$ The graphitization occurs in a multi-stage process, and is extremely dependent on sample cleanliness, pressure in the vacuum chamber etc. For these reasons, it is very difficult to obtain a uniform graphene coverage of less than 3 monolayers on a millimeter-scale $\mathrm{SiC}(001)$ sample. Therefore, for the transport measurements we used vicinal $\mathrm{SiC}(001) / \mathrm{Si}(001)$ samples with uniform trilayer graphene as proved by LEEM and ARPES data (Figures S2 and S5).

All STM experiments were conducted at RT with a single crystalline tungsten tips cleaned and sharpened in the UHV chamber. ${ }^{52} \mathrm{STM}$ topographic images were processed using WSxM software. ${ }^{53}$ ARPES experiments were performed with an ARPES $1^{2}$ endstation equipped with a Scienta R8000 hemispherical electron analyzer operated at the UE112-PGM2a beamline at BESSY-II. All ARPES measurements were performed at a photon energy of $62 \mathrm{eV}$ with a linear (mixed $s^{+} p$ ) light polarization. The beam spot size on the sample was about $0.4 \mathrm{~mm}$. Before ARPES characterization, the trilayer graphene/SiC(001) samples were annealed in the 
corresponding UHV preparation chambers at a variety of temperatures ranging from $200^{\circ} \mathrm{C}$ to $1000^{\circ} \mathrm{C}$ to remove possible contamination. SEC and valence band spectra of graphene synthesized on vicinal $\mathrm{SiC}(001)$ as a function of temperature in the range of $104-300 \mathrm{~K}$ were taken using the endstation of the Russian German Laboratory at BESSY-II. The LEEM and micro-LEED measurements were done using a SPELEEM microscope (Elmitec GmbH) installed on beamline I311 at the MAX-laboratory in Sweden.

The device with nano-gap contacts was fabricated by electron beam lithography (EBL) using single layer positive tone resist PMMA supplied by MicroChem Corp. After development, thick metal contacts consisting of $\mathrm{Ti}(5 \mathrm{~nm}) / \mathrm{Au}(45 \mathrm{~nm})$ were deposited by e-beam evaporation.

\section{Electron transport calculations}

We used the tight-binding approximation with a nearest neighbor hopping energy $\mathrm{t}(\mathrm{t}=2.7 \mathrm{eV})$ to describe the graphene system, ${ }^{54}$

$$
H=-t \sum_{i, j} C_{i}^{\dagger} C_{j}+\sum_{i} U_{i} C_{i}^{\dagger} C_{i}
$$

where $C_{i}^{\dagger}\left(C_{i}\right)$ creates (annihilates) an electron at site $i$ and $U_{i}$ is the on-site energy at site $i$. Sincea defect causes very small deviations in the bond length, ${ }^{55,56}$ we therefore propose that all of the hopping energiesare the same since all the carbon atoms are assumed to be equidistant.

In the electron transport simulation, we use nonequilibrium Green function (NEGF) theory and the Landauer-Keldysh method is used to calculate the transmission and I-V behaviors for theNBs in our system. ${ }^{41,42}$ The transmission function between the leads is evaluated by

$$
\mathrm{T}_{p q}=\operatorname{Tr}\left[\Gamma_{p} G^{R} \Gamma_{q} G^{A}\right]
$$


where $G^{R}\left(G^{A}\right)$ is the regular(advance) Green's function of the system. The lead broadening function $\Gamma_{p(q)}=i\left(\Sigma_{p(q)}-\Sigma_{p(q)}^{\mathrm{T}}\right)$, where $\Sigma_{\mathrm{p}(\mathrm{q})}$ is the self-energy of the lead. The conductance $\mathrm{G}(\mathrm{E})$ can be obtained from thetransimission function by $\mathrm{G}(\mathrm{E})=G_{0} T(E)$, where $G_{0}=\frac{2 e^{2}}{h}$ is the conductance quantum. Finally, the current can be obtained by $\mathrm{I}=\frac{2 e}{h} \int T(E)\left[f_{p}(E)-f_{q}(E)\right] d E$ where $f_{p(q)}$ is Fermi distrbution of each lead.

Conflict of Interest: The authors declare no competing financial interest.

Acknowledgment: This work was supported by Beijing Institute of Technology Research Fund Program for Young Scholars, Science Foundation Ireland (SFI) (No. 12/IA/1264), National Plan for Science and technology of KSU (Nos. NPST 1598-02, NPST 1466-02, NPST 2529-02), Russian Academy of Sciences, Russian Foundation for Basic Research (Nos. 11-02-01253, 1102-01256, 14-02-00949, 14-02-01234), SPP 1459 of the Deutsche Forschungsgemeinschaft, Marie Curie IIF grant within the 7th European Community Framework Programme, and the BMBF-Project No. 05K12GU2 and PSP-Element No. U4606BMB1211. We thank T. Chassagne, M. Zielinski and M. Portail (CRHEA-CNRS, Sophia Antipolis, France) for providing high quality $\mathrm{SiC}$ samples, B. Senkovskiy and D. Vyalikh for help with photoemission measurements, and A. Zakharov for help with LEEM/micro-LEED characterization.

\begin{abstract}
ASSOCIATED CONTENT
Supporting Information Available: Raman, LEEM, micro-LEED, STM, and ARPES characterization of the graphene/SiC(001) samples. This material is available free of charge via the Internet at http://pubs.acs.org.
\end{abstract}




\section{REFERENCES AND NOTES}

1. Craciun, M. F.; Russo, S.; Yamamoto, M.; Oostinga, J. B.; Morpurgo, A. F.; Tarucha S. Trilayer Graphene Is a Semimetal with AGate-Tunable Band Overlap. Nat. Nanotech. 2009, 4, 383-388.

2. Bao, W.; Jing, L.; Velasco, J.; Lee, Y.; Liu, G.; Tran, D.; Standley, B.; Aykol, M.; Cronin, S. B.; Smirnov, D.; et al. Stacking-Dependent Band Gap and Quantum Transport in Trilayer Graphene. Nat. Phys. 2011, 7, 948-952.

3. Lui, C. H.; Li, Z. Q.; Chen, Z. Y.; Klimov, P. V.; Brus, L. E.; Heinz, T. F. Image Stacking Order in Few-Layer Graphene. Nano Lett. 2011, 11,164-169.

4. Lui, C. H.; Li, Z. Q.; Mak, K. F.; Cappelluti, E.; Heinz, T. F. Observation of An Electrically TunableBand Gap in Trilayer Graphene. Nat. Phys. 2011, 7, 944-947.

5. Taychatanapat, T.; Watanabe, K.; Taniguchi, T.; Jarillo-Herrero, P. Quantum Hall Effect and Landau-Level Crossing of Dirac Fermions in Trilayer Graphene. Nat. Phys. 2011, 7, 621-625.

6. Zhang, F.; Jung, J.; Fiete, G. A.; Niu, Q.; MacDonald, A. H. Spontaneous Quantum Hall States in Chirally Stacked Few-Layer Graphene Systems. Phys. Rev. Lett. 2011, 106, 156801.

7. Zhang, L. Y.; Zhang, Y.; Camacho, J.; Khodas, M.; Zaliznyak, I. The Experimental Observation of Quantum Hall Effect of $\mathrm{I}=3$ Chiral Quasiparticles in Trilayer Graphene. Nat. Phys. 2011, 7, 953-957.

8. Castro Neto, A. H., Guinea, F., Peres, N. M. R., Novoselov, K. S.;Geim, A. K. The Electronic Properties of Graphene. Rev. Mod. Phys. 2009, 81, 109-162.

9. Bostwick, A.; Speck, F.; Seyller, T.; Horn, K.; Polini, M.; Asgari, R.; MacDonald, A. H.; Rotenberg, E. Observation of Plasmarons in Quasi-Freestanding Doped Graphene. Science 2010, 328, 999-1002. 
10. Novoselov, K. S.; Geim, A. K.; Morozov, S. V.; Jiang, D.; Katsnelson, M. I.; Grigorieva, I. V.; Dubonos, S. V.; Firsov, A. A. Two-Dimensional Gas of Massless Dirac Fermions in Graphene. Nature 2005, 438, 197-200.

11. Zhang, Y.; Tan, Y.-W.; Stormer, H. L.; Kim, P. Experimental Observation of the Quantum Hall Effect and Berry's Phase in Graphene. Nature 2005,438, 201-204.

12. Bolotin, K. I.; Sikes, K. J.; Jiang, Z.; Klima, M.; Fudenberg, G.; Hone, J.; Kim, P.; Stormer, H. L. Ultrahigh Electron Mobility in Suspended Graphene. Solid State Commun. 2008, 146, 351355.

13. Castro, E. V.; Novoselov, K. S.; Morozov, S. V.; Peres, N. M. R.; Lopes dos Santos, J. M. B.; Nilsson, J.; Guinea, F.; Geim, A. K.; Castro Neto, A. H. Biased Bilayer Graphene: Semiconductor with A Gap Tunable by the Electric Field Effect. Phys. Rev. Lett. 2007, 99, 216802.

14. Ohta, T.; Bostwick, A.; Seyller, T.; Horn, K.; Rotenberg, E. Controlling the Electronic Structure of Bilayer Graphene. Science 2006, 313, 951-954.

15. Zhang, Y. B.; Tang, T. T.; Girit, C.; Hao, Z.; Martin, M. C.; Zettl, A.; Crommie, M. F.; Shen, Y. R.; Wang, F. Direct Observation of AWidely Tunable Bandgap in Bilayer Graphene. Nature 2009, 459, 820 .

16. Mak, K. F.; Lui, C. H.; Shan, J.; Heinz, T. F. Observation of An Electric-Field-Induced Band Gap in Bilayer Graphene by Infrared Spectroscopy. Phys. Rev. Lett. 2009, 102, 256405.

17. Oostinga, J. B.; Heersche, H. B.; Liu, X. L.; Morpurgo, A. F.; Vandersypen, L. M. K. GateInduced Insulating State in Bilayer Graphene Devices. Nat. Mater. 2008, 7, 151-157.

18. Xia, F. N.; Farmer, D. B.; Lin, Y. M.; Avouris, P. Graphene Field-Effect Transistors with High On/Off Current Ratio and Large Transport Band Gap at Room Temperature. Nano Lett. 
2010, $10,715-718$.

19. Han, M. Y.; Ozyilmaz, B.; Zhang, Y.; Kim, P. Energy Band-Gap Engineering of Graphene Nanoribbons. Phys. Rev. Lett. 2007, 98, 206805.

20. Giesbers, A. J. M.; Peters, E. C.; Burghard, M.; Kern, K. Charge Transport Gap in Graphene Antidot Lattices, Phys. Rev. B 2012, 86, 045445.

21. Aoki, M.; Amawashi, H. Dependence of Band Structures on Stacking and Field in Layered Graphene. Solid State Commun. 2007,142, 123-127.

22. Avetisyan, A. A.; Partoens, B.; Peeters, F. M. Electric-Field Control of the Band Gap and Fermi Energy in Graphene Multilayers by Top and Back Gates. Phys. Rev. B 2009, 80, 195401.

23. Avetisyan, A. A.; Partoens, B.; Peeters, F. M. Electric Field Tuning of the Band Gap in Graphene Multilayers. Phys. Rev. B 2009, 79, 035421.

24. Zou, K.; Zhang, F.; Clapp, C.; MacDonald, A. H.; Zhu, J. Transport Studies of Dual-Gated ABC and ABA Trilayer Graphene: Band Gap Opening and Band Structure Tuning in Very Large Perpendicular Electric Fields. Nano Lett. 2013, 13, 369-373.

25. Chen, C. X.; Wu, J. Z.; Lam, K. T.; Hong, G. S.; Gong, M.; Zhang, B.; Lu, Y.; Antaris, A. L.; Diao, S.;Guo, J.; et al. Graphene Nanoribbons Under Mechanical Strain. Adv. Mater. 2014, 27, 303-309.

26. Kim, K. S.; Walter, A. L.; Moreschini, L.; Seyller, T.; Horn, K.; Rotenberg, E.; Bostwick, A. Coexisting Massive and Massless Dirac Fermions in Symmetry-Broken Bilayer Graphene. Nat.Mater. 2013, 12, 887-892.

27. Li, X.;Cai, W.; An, J.; Kim, S.; Nah, J.; Yang, D.; Piner, R.; Velamakanni, A.; Jung, I.;

Tutuc, E.; et al. Large-Area Synthesis of High-Quality and Uniform Graphene Films on Copper Foils. Science 2009, 324, 1312-1314. 
28. Bae, S.; Kim, H.; Lee, Y.; Xu, X.; Park, J.-S.; Zheng, Y.; Balakrishnan, J.; Lei, T.; Kim, H. R.; Song, Y. I.; et al. Roll-to-Roll Production of 30-Inch Graphene Films for Transparent Electrodes. Nat. Nanotech. 2010, 5, 574-578.

29. Berger, C.; Song, Z.; Li, T.; Li, X.; Ogbazghi, A. Y.; Feng, R.; Dai, Z., Marchenkov, A. N.; Conrad, E. H.; First, P. N.; et al. Ultrathin Epitaxial Graphite:2D Electron Gas Properties and A Route Toward Graphene-Based Nanoelectronics. J. Phys. Chem. B 2004,108, 19912-19916.

30. Chaika, A. N.; Molodtsova, O. V.; Zakharov, A. A.; Marchenko, D.; Sánchez-Barriga, J.; Varykhalov, A.; Shvets, I. V.; Aristov V. Y. Continuous Wafer-Scale Graphene on CubicSiC(001), Nano Research, 2013, 6, 562-570.

31. Chaika, A.N.; Molodtsova, O. V.; Zakharov, A. A.; Marchenko, D.; Sánchez-Barriga, J.; Varykhalov, A.; Babenkov, S. V.; Portail, M.; Zielinski, M.; Murphy, B. E.; et al. Rotated Domain Network in Graphene on Cubic-SiC(001). Nanotech. 2014, 25, 135605.

32. Aristov, V. Y.; Urbanik, G.; Kummer, K.; Vyalikh, D. V.;Molodtsova, O. V.; Preobrajenski, A. B.; Zakharov, A. A.;Hess, C.; Hänke, T.; Büchner, B.; et al. Graphene Synthesis on Cubic $\mathrm{SiC} / \mathrm{Si}$ Wafers. Perspectives for Mass Production of Graphene-Based Electronic Devices. Nano Lett. 2010, 10, 992-995.

33. Huang, P. Y.; Ruiz-Vargas, C. S.; van der Zande, A. M.; Whitney, W. S.; Levendorf, M. P.; Kevek, J. W.; Garg, S.; Alden, J. S.; Hustedt, C. J.; Zhu, Y.; et al. Grains and Grain Boundaries in Single-Layer Graphene Atomic Patchwork Quilts. Nature 2011, 469, 389-392.

34. Kim, K.; Lee, Z.; Regan, W.; Kisielowski, C.; Crommie, M. F.; Zettl, A. Grain Boundary Mapping in Polycrystalline Graphene. ACS Nano 2011, 5, 2142-2146.

35. Tsen, A. W.; Brown, L.; Levendorf, M. P.; Ghahari, F.; Huang, P. Y.; Havener, R. W.; RuizVargas, C. S.; Muller, D. A.; Kim, P.; Park, J. Tailoring Electrical Transport Across Grain 
Boundaries in Polycrystalline Graphene. Science 2012, 336, 1143-1146.

36. Yazyev, O. V.;Louie, S. G. Electronic Transport in Polycrystalline Graphene. Nat. Mater. 2010, 9, 806-809.

37. Menezes, M. G.;Capaz, R. B.; Louie, S. G. Ab InitioQuasiparticle Band Structure of ABA and ABC-Stacked Graphene Trilayers. Phys. Rev. B 2014, 89, 035431.

38. Marchenko, D.; Varykhalov, A.; Scholz, M. R.; Sánchez-Barriga, J.; Rader, O.; Rybkina, A.; Shikin, A. M.; Seyller, Th.; Bihlmayer, G. Spin-Resolved Photoemission and Ab InitioTheory of Graphene/SiC. Phys. Rev. B2013, 88, 075422.

39. Marchenko, D.; Sánchez-Barriga, J.; Scholz, M. R.; Rader, O.; Varykhalov, A. Spin Splitting of Dirac Fermions in Aligned and Rotated Graphene on $\operatorname{Ir}(111)$. Phys. Rev. B 2013, 87, 115426.

40. Marchenko, D.; Varykhalov, A.; Scholz, M. R.; Bihlmayer, G.; Rashba, E.I.; Rybkin, A.; Shikin, A.M.; Rader, O. Giant Rashba Splitting in Graphene due to Hybridization with Gold. Nat. Commun. 2012, 3, 1232.

41. Ferry, D. K.; Goodnick, S. M. Transport in Nanostructures. Cambridge, UK, 1997.

42. Datta, S. Electronic Transport in Mesoscopic Systems. Cambridge University Press, Cambridge, 1995.

43. Banhart, F.; Kotakoski, J.; Krasheninnikov, A. V. Structural Defects in Graphene. ACS Nano 2011, 5, 26-41.

44. Thrower, P. A.; Mayer, R. M. Point Defects and Self-Diffusion in Graphite. Phys. Stat. Sol. (a) 1978, 47, 11-37.

45. Suarez-Martinez, I.; El-Barbary, A. A.; Savini, G.; Heggie, M. I. First-Principles Simulations of Boron Diffusion in Graphite. Phys. Rev. Lett. 2007, 98, 015501.

46. Ma, Y. Simulation of Interstitial Diffusion in Graphite. Phys. Rev. B 2007, 76, 075419. 
47.Trevethan, T.; Latham, C. D.; Heggie, M. I.; Briddon, P. R.; Rayson, M. J. Vacancy Diffusion and Coalescence in Graphene Directed by Defect Strain Fields. Nanoscale 2014, 6, 2978-2986.

48. Goldberg Yu.; Levinshtein M.E.; Rumyantsev S.L. Properties of Advanced Semiconductor Materials GaN, AlN, SiC, BN, SiC, SiGe. Eds. Levinshtein M.E., Rumyantsev S.L., Shur M.S., John Wiley \& Sons, Inc., New York, 2001, 93-148.

49. Yoon, D.; Son, Y. -W.; Cheong, H. Negative Thermal Expansion Coefficient of Graphene Measured by Raman Spectroscopy. Nano Lett. 2011, 11, 3227-3231.

50. Gao, W.; Huang, R. Thermomechanics of Monolayer Graphene: Rippling, Thermal Expansion and Elasticity. J. Mecha. .Phys. Sol. 2014, 66, 42-58.

51. Bao, W.; Miao, F.; Chen, Z.; Zhang, H.; Jang, W.; Dames, C.; Lau, C. N. Controlled Ripple Texturing of Suspended Graphene and Ultrathin Graphite Membranes. Nat. Nanotech. 2009, 4, $562-566$.

52. Chaika, A. N.; Orlova, N. N.; Semenov, V. N.; Postnova, E. Y.; Krasnikov, S. A.; Lazarev, M. G.; Chekmazov, S. V.; Aristov, V. Y.; Glebovsky, V. G.; Bozhko, S. I.; et al. Fabrication of [001]-Oriented Tungsten Tips for High Resolution Scanning Tunneling Microscopy. Sci. Rep. $\underline{\mathbf{2 0 1 4}, 4,3742 .}$

53. Horcas, I.; Fernández, R.; Gómez-Rodriguez, J. M.; Colchero, J.; Gómez-Herrero, J.; Baro, A. M. WSXM: A Software for Scanning Probe Microscopy and A Tool for Nanotechnology. Rev. Sci. Instrum. 2007, 78, 013705.

54. Bahamon, D. A.; Pereira, A. L. C.; Schulz, P. A. Third Edge for AGraphene Nanoribbon: A Tight-Binding Model Calculation. Phys. Rev. B 2011, 83, 155436.

55. Yazyev, O. V.; Louie, S. G. Topological Defects in Graphene: Dislocations and Grain Boundaries. Phys. Rev. B 2010, 81, 195420. 
56. Appelhans, D. J.; Carr, L. D.; Lusk, M. T. Embedded Ribbons of Graphene Allotropes: An Extended Defect Perspective. New J. Phys. 2010, 12, 125006. 
Figures and captions

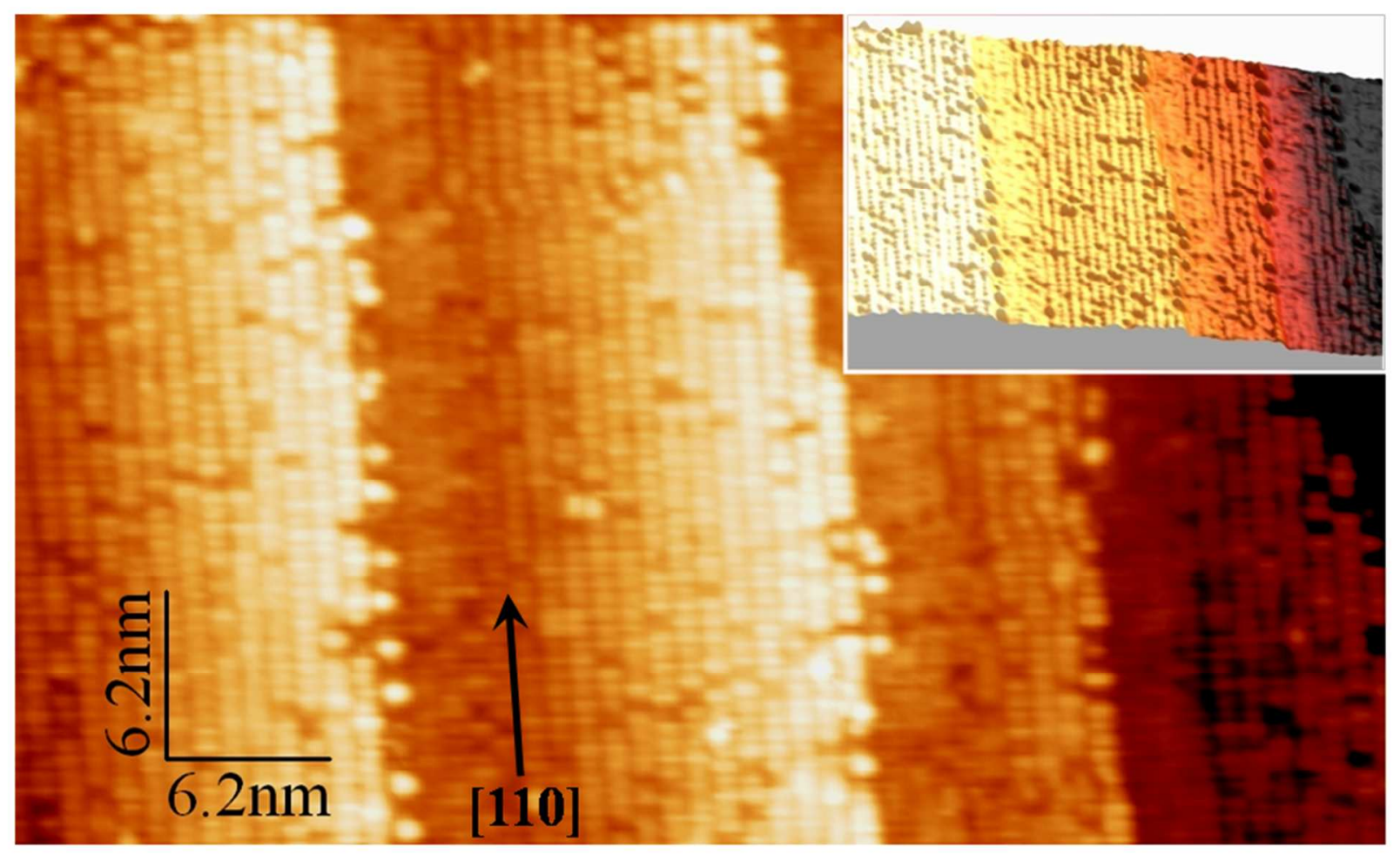

Figure 1 | STM characterization of the vicinal SiC(001). 2D- and 3D-representations (inset) of a $51 \times 31 \mathrm{~nm}^{2}$ atomically resolved STM image of the $\mathrm{SiC}(001) 3 \times 2$ reconstruction. The image demonstrates that the step direction is close to the [110] direction of the SiC crystal lattice. The image was measured at $\mathrm{U}=-2.3 \mathrm{~V}$ and $\mathrm{I}=80 \mathrm{pA}$. 

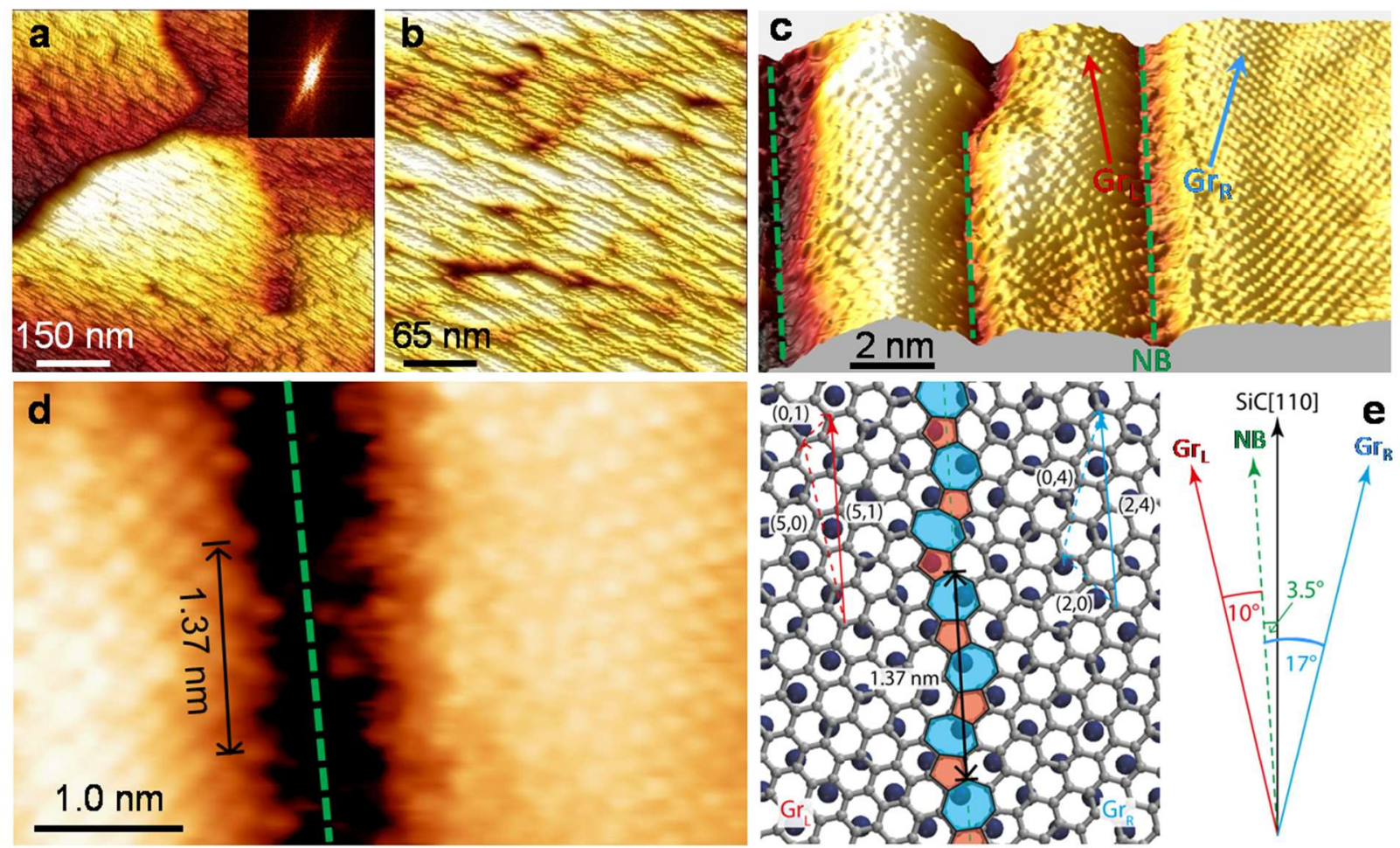

Figure 2| STM characterization of the graphene grown on vicinal $\mathrm{SiC}(001)$. (a) and (b) Large area STM images of graphene nanoribbons synthesized on the vicinal $\mathrm{SiC}(001)$. Inset in panel (a) shows the Fast Fourier Transform of the STM image proving one preferential direction of the NBs on the vicinal sample. (c) and (d) Atomically resolved STM images of graphene nanoribbons showing the system of domains rotated $17^{\circ}$ clockwise $\left(\mathrm{Gr}_{\mathrm{R}}\right)$ and $10^{\circ}$ anticlockwise $\left(\mathrm{Gr}_{\mathrm{L}}\right)$ relative to the NB which is rotated $3.5^{\circ}$ anticlockwise from the [110] direction (c) and the atomic structure of the NB (d). The images were measured at $\mathrm{U}=-100 \mathrm{mV}, \mathrm{I}=68 \mathrm{pA}$.(e) Schematic model of the NB for the asymmetrically rotated nanodomains in panels (c) and (d). For the angles shown a periodic structure of distorted pentagons and heptagons is formed. 

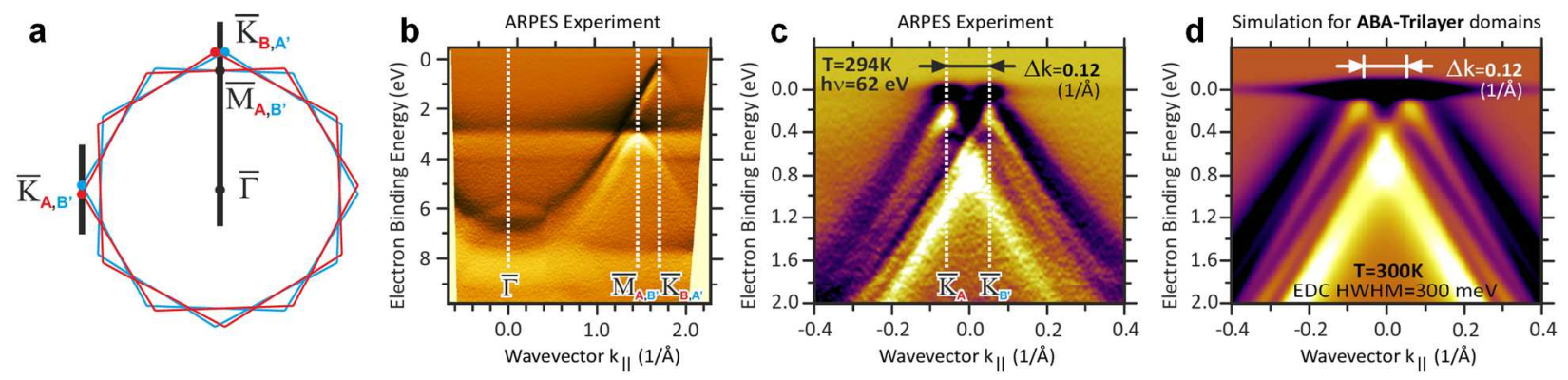

Figure 3| ARPES characterization determining the electronic structure and the Bernal stacking nature of the trilayer graphene on the vicinal $\mathrm{SiC}(001)$. (a) Effective surface Brillouin zone corresponding to four rotated domain variants. (b) and (c) Dispersion of the $\pi$-band in the graphene along the directions indicated in panel (a). (d) Simulations for ABA-stacked trilayer graphene with a high density of nanodomain boundaries. 

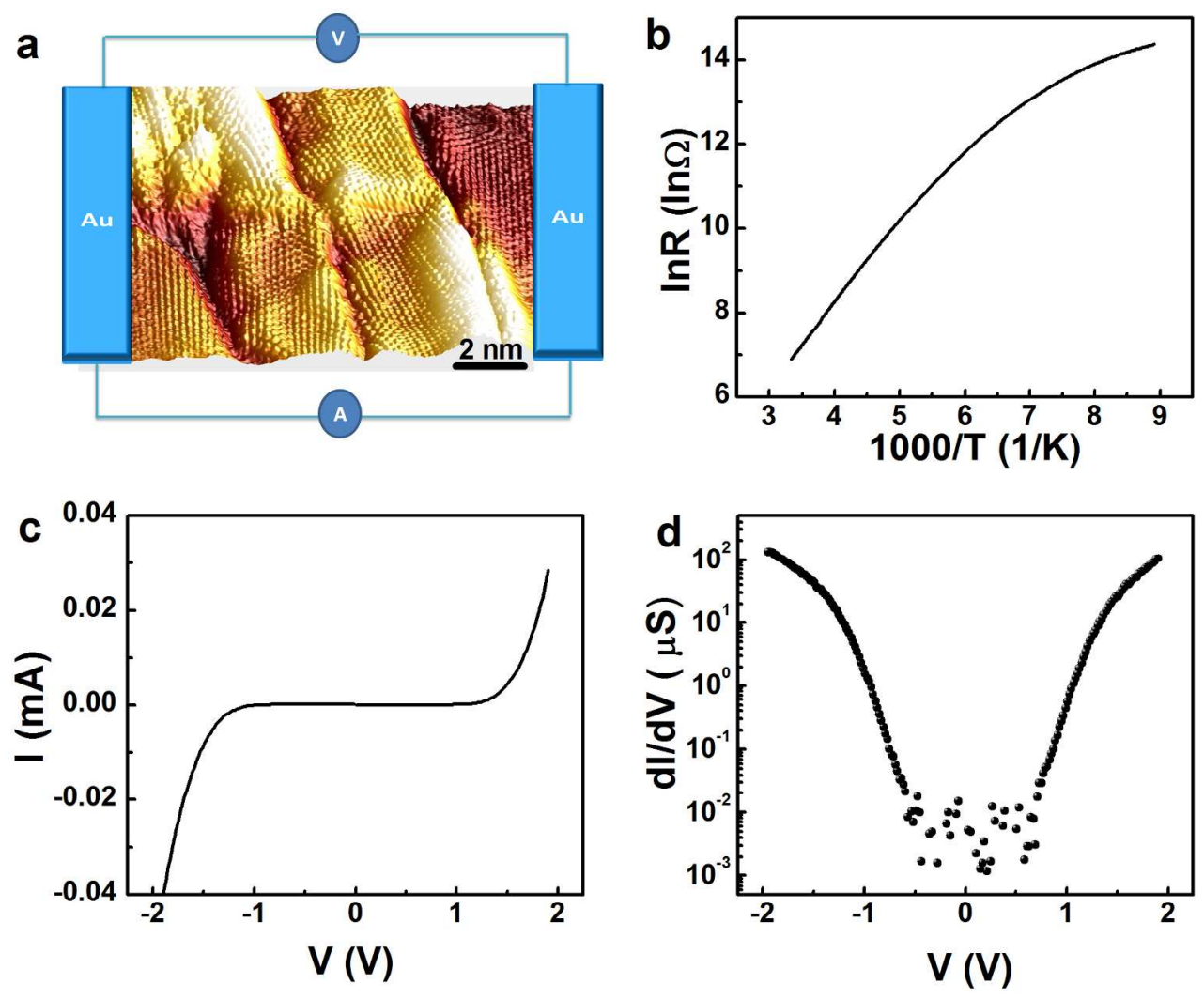

Figure 4| Electrical detection of the opening of a transport gap in Bernal stacked trilayer graphene on a vicinal $\mathrm{SiC}$ substrate. (a) Schematic drawing of the nano-gap device. (b) R-T curve measured with a bias voltage of $0.5 \mathrm{~V}$. (c) I-V curve measured at $10 \mathrm{~K}$ and (d) the corresponding $\mathrm{dI} / \mathrm{dV}$ curve to demonstrate the existence of a transport gap. 
2

3

4

5

6

7

8

9

10

11

12

13

14

15

16

17

18

19

20

21

22

23

24

25

26

27

28

29

30

31

32

33

34

35

36

37

38

39

40

41

42

43

44

45

46

47

48

49

50

51

52

53

54

55

56

57

58

59

60
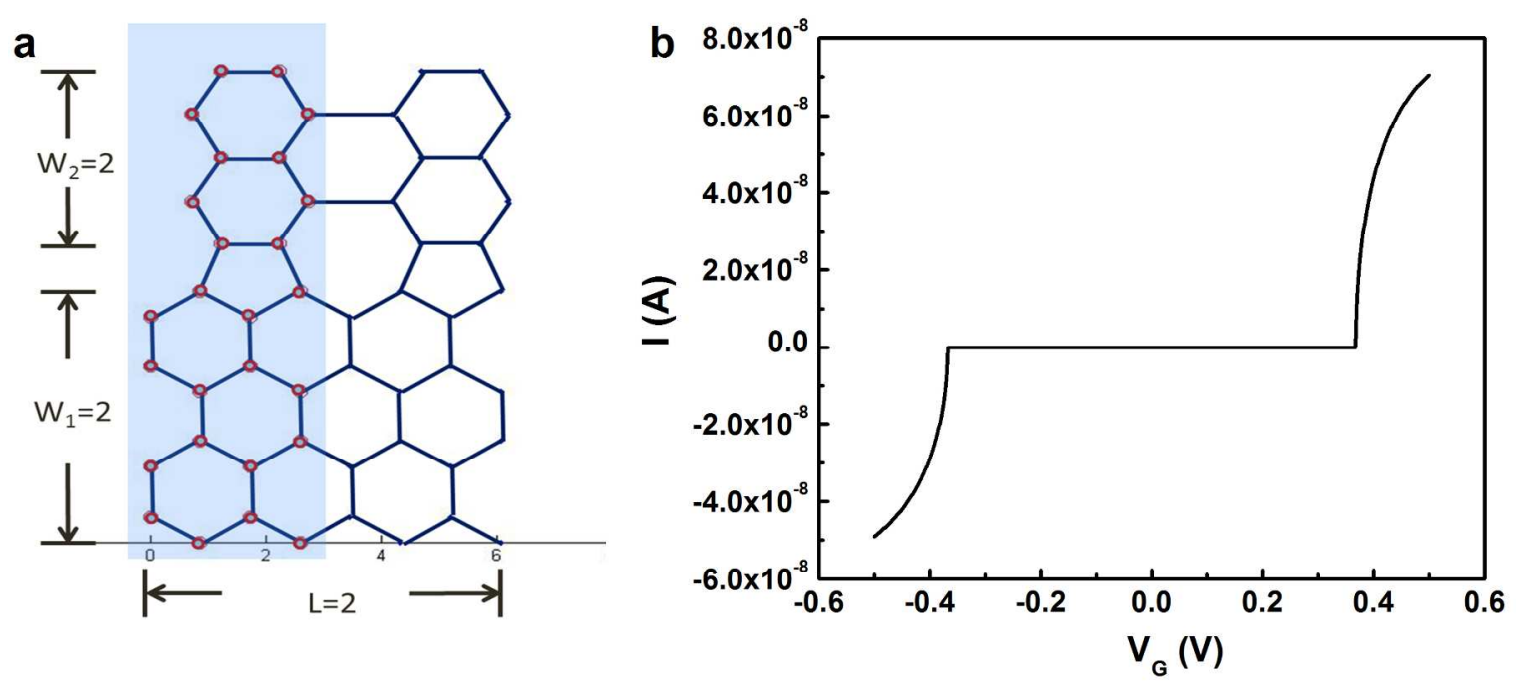

Figure 5| Current as a function of gate voltage. (a) Schematic drawing of the model used, where $\mathrm{L}$ is the length of the NB, $\mathrm{W}_{1}$ is the width of armchair structure and $\mathrm{W}_{2}$ is the width of zigzag structure. (b) Current as a function of back voltage calculated from first-principle simulation. 

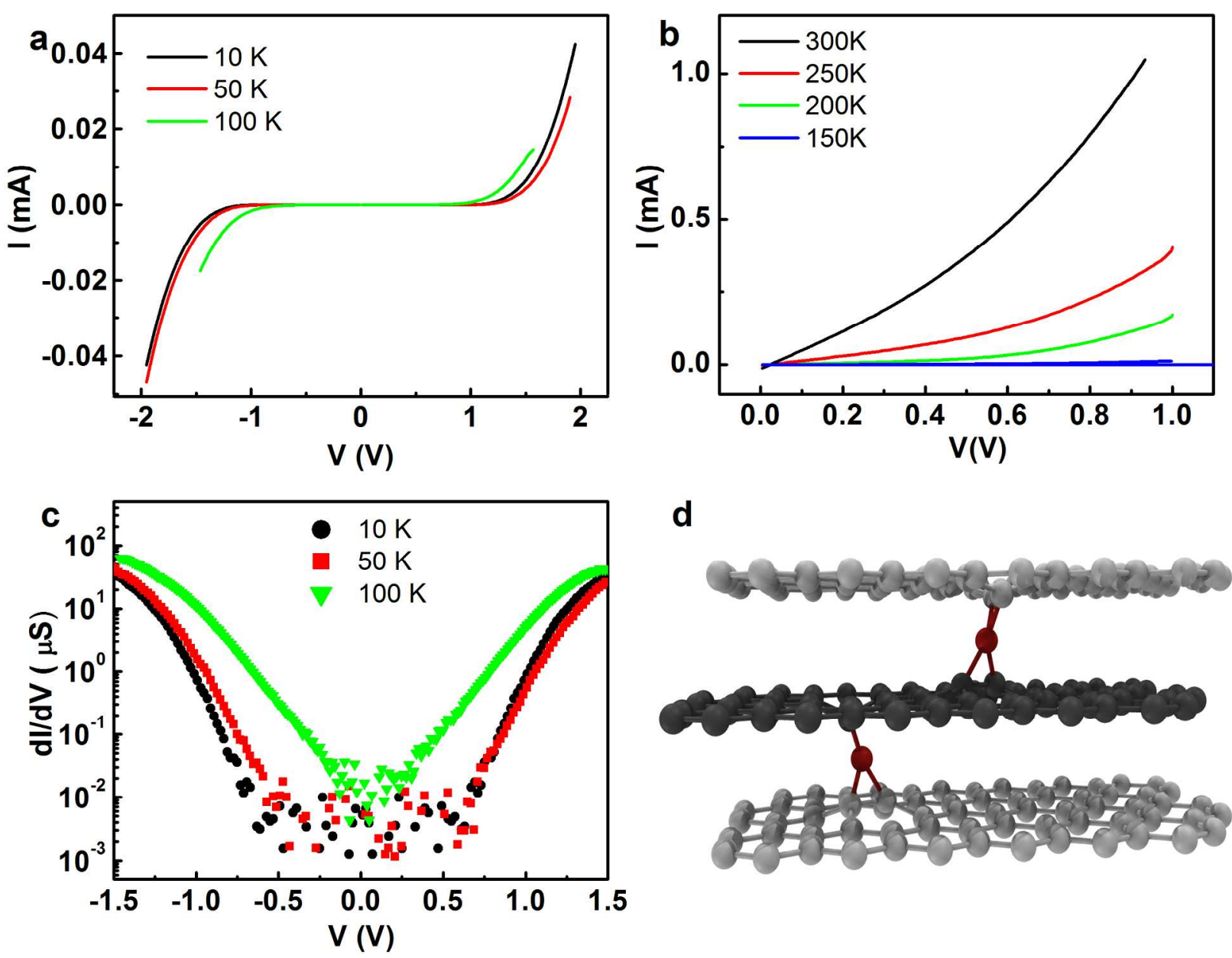

d

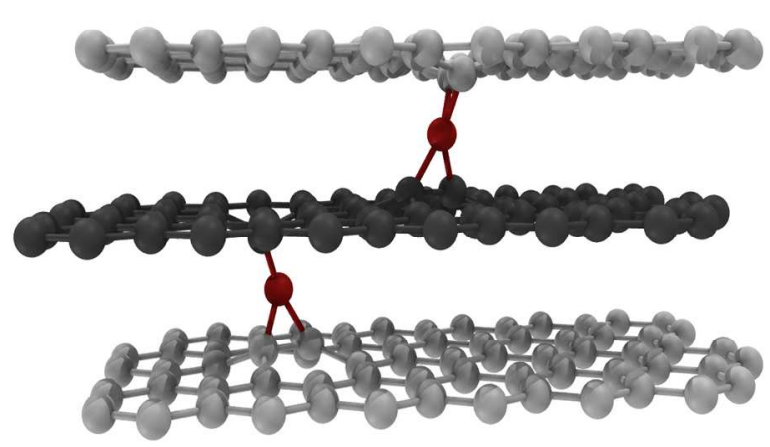

Figure 6| I-V curves measured at different temperatures with current across the self-aligned NBs.

(a) I-V curves measured at $10 \mathrm{~K}, 50 \mathrm{~K}$, and $100 \mathrm{~K}$. (b) I-V curves measured at $150 \mathrm{~K}, 200 \mathrm{~K}, 250$

$\mathrm{K}$ and $300 \mathrm{~K}$. (c) Corresponding $\mathrm{dI} / \mathrm{dV}$ curves for temperatures below $150 \mathrm{~K}$. (d) Schematic drawing of interstitial defects in trilayer graphene. 

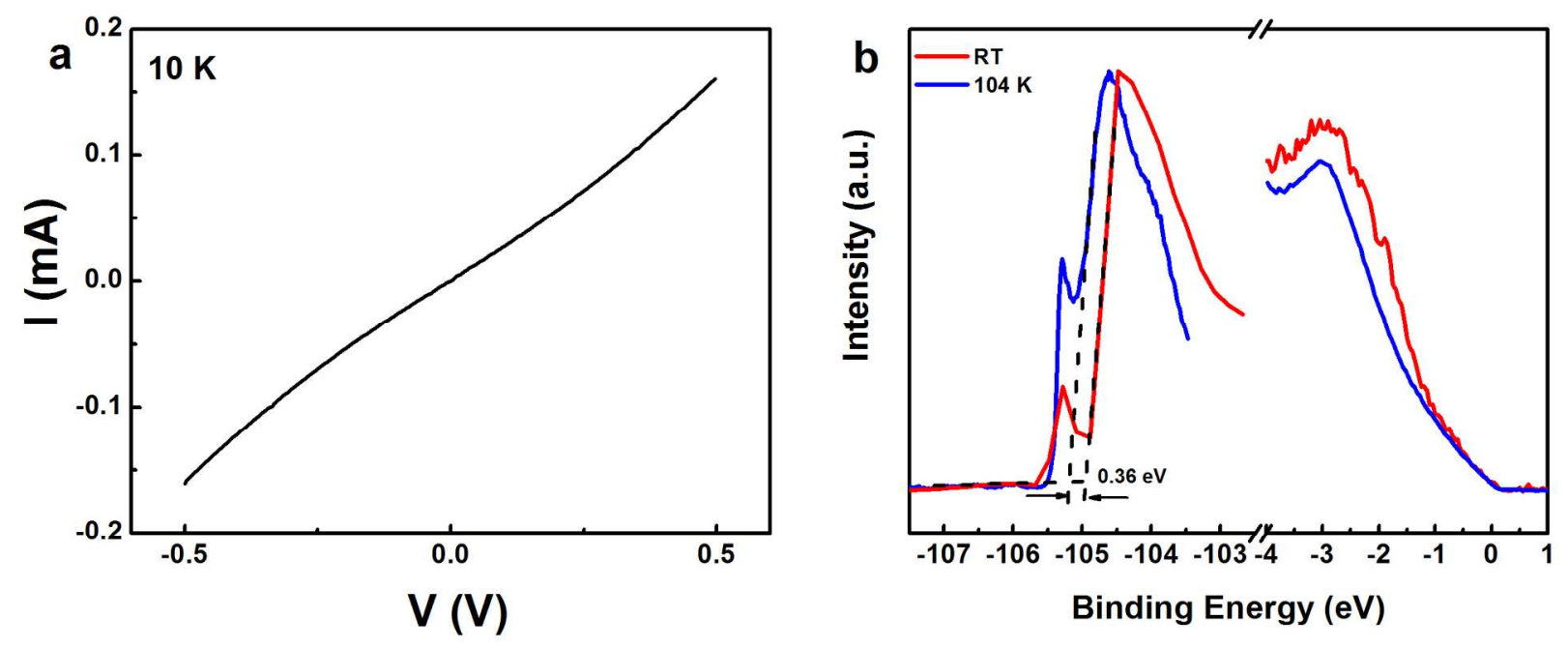

Figure 7| I-V curve measured with current applied along the NBs and photoemission measurements at different temperatures. (a) I-V curve measured at $10 \mathrm{~K}$ along NBs. (b) SEC and valence band edge taken at 104 and $300 \mathrm{~K}$ at a photon energy of $110 \mathrm{eV}$. The sample was biased at -18.2 and $-8.42 \mathrm{~V}$ at 104 and $300 \mathrm{~K}$, respectively. 